\title{
Ulcerative Paraneoplastic Dermatomyositis in the Setting of Positive Transcriptional Intermediary Factor $1-\gamma$ Antibody
}

Mina Z. Al Awqati@, MD, Division of Rheumatology, Mayo Clinic; Jason C. Sluzevich $₫$, MD, Department of Dermatology, Mayo Clinic;

Florentina Berianu (®, MD, Division of Rheumatology, Mayo Clinic, Jacksonville, Florida, USA. Address correspondence to Dr. F. Berianu, Division of Rheumatology, Mayo Clinic, 4500 San Pablo Rd., Jacksonville, FL 32224, USA. Email: Berianu.Florentina@mayo.edu. The authors declare no conflicts of interest. Patient consent was obtained. Institutional review board approval was not required according to the authors' institution.

Dermatomyositis (DM) is an autoimmune inflammatory disease of the skin with or without muscle involvement ${ }^{1}$. One-third of patients present with malignant disease within 3 years ${ }^{2}$.

A 60-year-old woman with a remote history of ovarian cancer presented with a 1-month history of severe dysphagia, symmetric proximal muscle weakness, and a progressive painful ulcerative skin eruption associated with her second recurrence of metastatic

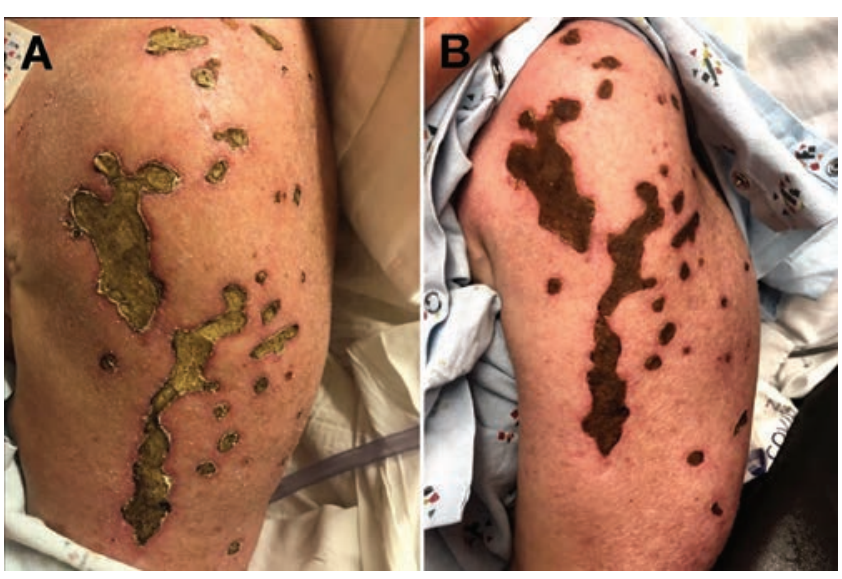

Figure 1. (A) Multiple well-defined geographic peripheral erythematous ulcers with adherent crust. (B) Ulcer expansion arrested after immunosuppressive therapy.

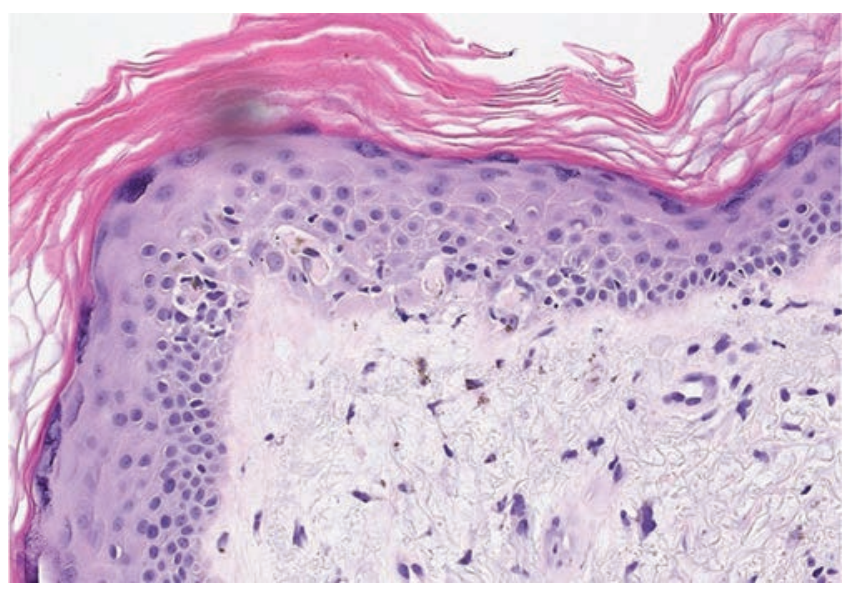

Figure 2. Extensive vacuolar interface dermatitis with focal epidermal dyskeratosis. H\&E stain, $40 \times$ magnification. ovarian cancer treated 4 years ago. Physical examination revealed 4/5 proximal muscle weakness, Gottron papules, heliotrope rash, and multiple irregular ulcers with overlying eschars (Figure 1A). Serum creatine kinase was normal, while liver function tests and aldolase were mildly elevated. Serology showed positive transcriptional intermediary factor $1-\gamma($ TIF $1-\gamma)$ and negative melanoma differentiation-associated protein 5 (MDA-5) antibodies. Electromyography confirmed proximal myopathy. Skin biopsy revealed interface dermatitis with underlying dermal mucinosis and pigment incontinence (Figure 2). High-dose pulse corticosteroids and intravenous Ig improved the myositis and arrested the spread of the ulcers (Figure 1B).

TIF $1-\gamma$ antibodies are associated with a DM phenotype of myositis and extensive skin involvement that may include classic heliotropic erythema, psoriasiform plaques, or near erythroderma. Ulcerated and eroded skin lesions can be seen with anti-MDA-5 DM, often in association with interstitial lung disease; they are also a well-described finding in paraneoplastic DM. Paradoxically, despite the high risk of malignancy in older patients with TIF1- $\gamma$ autoantibodies, skin ulceration is phenotypically uncommon in comparison with classic adult-onset cancer-associated DM presentations ${ }^{3,4,5}$.

\section{REFERENCES}

1. Masiak A, Kulczycka J, Czuszyńska Z, Zdrojewski Z. Clinical characteristics of patients with anti-TIF1- $\gamma$ antibodies. Reumatologia 2016;54:14-8.

2. Oldroyd A, Sergeant JC, New P, McHugh NJ, Betteridge Z, Lamb JA, et al. The temporal relationship between cancer and adult onset anti-transcriptional intermediary factor 1 antibody-positive dermatomyositis. Rheumatology 2019;58:650-5.

3. Hall JC, Casciola-Rosen L, Samedy LA, Werner J, Owoyemi K, Danoff SK, et al. Anti-MDA 5-associated dermatomyositis: expanding the clinical spectrum. Arthritis Care Res 2013; 65:1307-15.

4. Moghadam-Kia S, Oddis CV, Sato S, Kuwana M, Aggarwal R. Anti-melanoma differentiation-associated gene 5 is associated with rapidly progressive lung disease and poor survival in US patients with amyopathic and myopathic dermatomyositis. Arthritis Care Res 2016;68:689-94.

5. Betteridge Z, McHugh N. Myositis-specific autoantibodies: an important tool to support diagnosis of myositis. J Intern Med 2016;280:8-23. 